\title{
Differential diagnosis between occupational chronic intoxication with organic solvents and ethanol abuse by biological markers. Case report and literature review
}

${ }^{1}$ Carol Davila University of Medicine and Pharmacy, Bucharest, Romania

${ }^{2}$ Faculty of Medicine, University "Ovidius" of Constanta

\begin{abstract}
Given the occupational exposure to substances contained in petroleum heated to high temperature, cholestatic liver disease, neurological signs and symptoms can occur. We present a case report of a male patient with no tobacco exposure or alcohol use, but with prolonged occupational exposure to petroleum vapours and natural gases like methane, propane butane who developed liver and neurologic disease, in the absence of protective equipment. Delayed diagnosis was established after 9 years of the first symptoms occurrence. Differential diagnosis with alcoholic etiology was considered and biological biomarkers were useful.
\end{abstract}

Keywords: biomarkers, alcohol, liver disease, petroleum solvents

\section{Oana Arghir}

Faculty of Medicine, University "Ovidius" of Constanta University Street, No. 1, Campus B, Constanta, Romania

email : arghir_oana@yahoo.com

\section{Introduction}

Petroleum contains around 17,000 organic compounds, the most important being aliphatic non halogenated hydrocarbons, aromatic volatile compounds (benzene, toluene). The route of entrance for all these toxics, together called organic solvents, was mostly through skin contact and inhalation. Aromatic compounds are liquid at normal temperatures, but can fairly evaporate reaching significant concentrations in the air from temperatures of $20^{\circ} \mathrm{C}$ [1]. Fatty liver disease and toxic hepatitis were described linked to organic solvent exposure [2,3]. Hepatic ultrasonic changes occurred in individuals exposed to perchloroethylene during work time [4], and even fatal fulminant hepatic failure can be induced by solvents [5]. Health risk of workers associated with crude oil spill exposure was described in literature [6]. So, initial motivation for reporting this case consisted in a male patient with no tobacco exposure or alcohol use, with prolonged occupational exposure to petroleum vapours and 
natural gases like methane, propane butane in the absence of protective equipment who developed liver and neurologic disease.

\section{Ease report.}

A 51 year-old, non-smoking male patient, with a prolonged occupational history of 28 years, worked in the last 25 years as an extraction operator in an oil company. Occupational interview revealed chronic exposure to organic solvents contained in the petroleum (aliphatic solvents, superior and inferior aromatic solvents, naphtha solvents). He took samples of petroleum, heated to $40-65^{\circ} \mathrm{C}$, in order to be analysed and to establish the substances he needs to add manually to the tank for the optimal separation of the fractions of petroleum.

\section{Clinical and paraclinical presentation.}

In 2007, started neurologic symptoms as glove and sock paraesthesia. One year later, in 2008, patient developed walking and equilibrium disorders with progressive worsening. In 2011, the patient was admitted at Neurology Department of "Bagdasar" Hospital of Bucharest, Romania and initial diagnosis of disc hernia was set. This diagnosis was later refuted by the CT scan of the thoracic and lumbar spine. Subsequent hospitalization was done in Targu Mures Neurology Clinic, and neuropathy and chronic cholestatic hepatitis were found. A possible occupational aetiology was considered and patient have changed his workplace. In 2012, he was firstly admitted in Occupational Medicine Clinic of Colentina Clinical Hospital of Bucharest for recurrent symptoms of sock and glove paraesthesia, walking disorder, equilibrium disorder, and new manifestations as marked physical asthenia, biliary dyspepsia, anorexia and arthralgia. Physical exam revealed a large abdomen, hepatomegaly (anterior hepatic diameter of $14 \mathrm{~cm}$ ). Several investigations were performed:
Blood tests revealed hepatic cytolysis, cholestasis, hypoalbuminemia (see Table 1) and negative markers for viral hepatitis.

The electromyography (EMG) showed a predominantly sensitive chronic axonal polyneuropathy, compatible with an organic solvent exposure effect.

Abdominal echography confirmed the hepatomegaly and showed a nonhomogeneous structure, with hypoechogenic areas and a small quantity of ascites liquid.

Upper gastrointestinal endoscopy (UGE) revealed second degree of oesophageal varices.

Differential diagnosis with alcoholic liver disease, viral hepatitis and Wilson disease was done. Alcohol consumption was denied by both patient and his family. Wilson disease was excluded. Positive diagnosis consisted in hepatic cirrhosis following chronic hepatitis, cholestatic form; portal hypertensive gastropathy with oesophageal varices.

Prescribed therapy included beta blocker, oral non absorbable antibiotic, B-complex vitamins. Retirement was proposed for medical reasons.

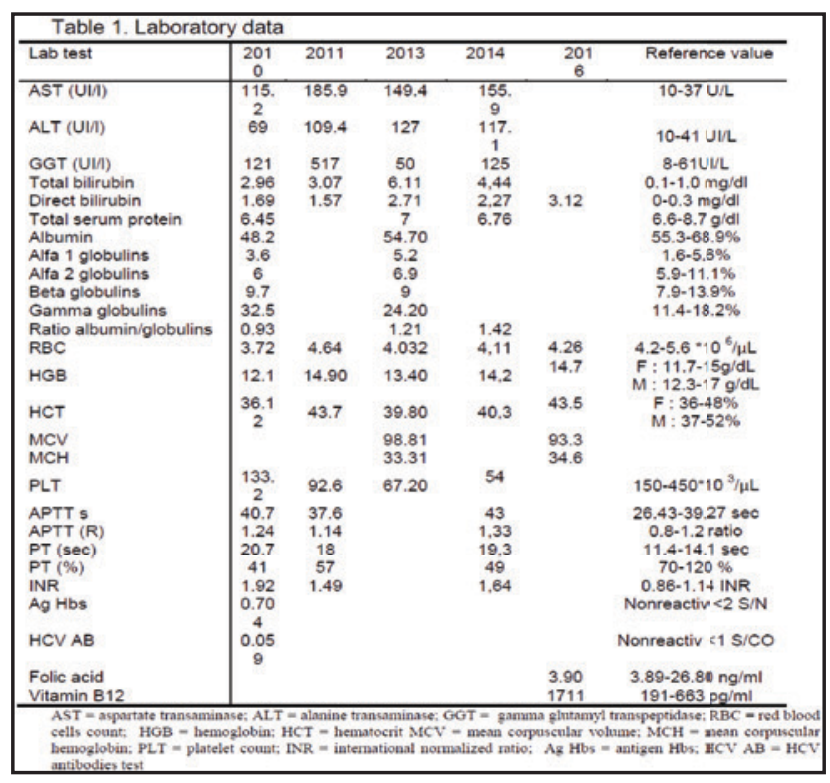

Evolution. Patient medical condition subsequently worsened for both neurological (walking with crutches), and liver disease (clinical and biological signs of hepatic insufficiency). In 
2016, he underwent a second UGE which showed a third degree of oesophageal varices, without signs of severity, severe portal hypertensive gastropathy with "snake skin" appearance of the mucosa and intramucosal haemorrhages. The ecographic examination revealed liver dimensions of $136 / 72 \mathrm{~mm}$, with a non-homogeneous micronodular structure; the cholecyst was distended, double-curved, with residue, but no apparent calculi; the portal vein diameter was $12.3 \mathrm{~mm}$ and splenic vein diameter 7.9 $\mathrm{mm}$; the main biliary tract was normal; the pancreas was hyperechogenic; the spleen long axis was 138/71 $\mathrm{mm}$ with slightly enlarged veins of the hilum (portal hypertension); both kidneys were of normal size, normal parenchymal index, no calculi. The Fibroscan median result was $36.8 \mathrm{kPa}$ corresponding to stage $\mathrm{F} 4$ (cirrhosis or advanced fibrosis).

According to occupational exposure, final diagnosis was chronic occupational intoxication with organic solvents, toxic hepatic cirrhosis secondary and toxic peripheral sensory-motor polyneuropathy.

\section{Comments.}

Chronic toxic occupational hepatitis develops either from prolonged exposure to small quantities of a toxic substance, or as an unfavourable evolution of an acute or subacute hepatitis. There are three conditions to be met in order to confirm the diagnosis of toxic occupational hepatitis: (1) the liver disease should follow the exposure to a hepatotoxic substance (or mixture of substances) present at the workplace; (2) the transaminases should be at least two times higher than the upper limit of normal; (3) other causes of hepatitis should be excluded, such as infectious factors, medication use (like contraceptives, statins, aspirin, tetracycline, methotrexate), alcohol consumption or voluntary inhalation of solvents [3]. The traditional biomarkers of chronic alcohol consumption, used frequently in the clinic (mean corpuscular volume [MCV], ALT, AST and GGT) are modified in the same manner both in the chronic alcoholic hepatitis and in occupational hepatitis caused by exposure to hydrocarbons. Occupational exposure in a refinery remained significantly associated with a significant increase in the elevation of GGT and ALT even after correcting for obesity and alcohol consumption [7]. GGT is also increased in cholestasis and cholestatic adverse effects of aromatic hydrocarbons (elevation of GGT and alkaline phosphatase) have been described as well [8].

Another difficulty of differential diagnosis is that most markers of alcohol consumption, as well as those of exposure to organic solvents, have relatively a short half-life, being present in the biological fluids for short periods of time. All the described markers (GGT, Carbohydrate-deficient transferrin [CDT] [8], ethylglucuronide, 5- hydroxytryptophol [5-HTOL], sialic acid, hexosaminidase, fatty acid ethyl esters) may have normal values following 4-5 weeks of abstinence $[10,11,12]$, and, thus, they are useful only in acute alcohol ingestion, or in monitoring the abstinence. [13] Similarly, 1-2 months after cessation of occupational exposure there are no high levels of hippuric acid, catechols, hydroquinones or muconic acid in urine. Our patient was firstly admitted in an occupational clinic a long time after occupational exposure stopped, and any attempt to use biomarkers was not applicable. He doesn't recall an acute intoxication, but was chronically exposed for 25 years to aromatic non halogenated volatile compounds and oil well gases, substances that can cause hepatic and neurological lesions. There are no studies evaluating the influence of organic solvents exposure on these markers, therefore their usage is of limited value. However, what we do know is that oxidative stress markers in blood (superoxide dismutase, glutathione peroxidase etc.) persist at high levels even after 6 months of alcohol abstinence [14].

Protective equipment used by patient was purely designed to avoid the consequences of injuries (helmet, cords), but did not stop the absorption through skin or airways by inhalation because it lacked protective gloves, or respiratory mask. The interactions among different organic solvents are generally shown to enhance their action $[15,16,17]$. An additive effect of alcohol was described in the case of occupational exposure to organic solvents, increasing the hepatic clearance of chlorinated 
compounds and enhances the destructive effects of that occupational toxic [18]. Alcohol, also, induces bronchial epithelium damage and increases airways absorption of solvents. Ingested alcohol is metabolised in the liver, but a certain quantity might escape the first-passage metabolization and reaches the alveoli. Bronchial epithelial cells contain activated P450 E21 cytochrome and degrades alcohol under the same pathways as the hepatic cytochrome, generating acetaldehyde and reactive oxygen species. Oxidative stress and lipid peroxidation alter the protection barrier of the bronchial epithelium increasing the absorption of toxics [19]. An attempt to quantify chronic consumption of alcohol is by revealing persistent markers in the red blood cells or in the hair. MCV increases through direct medullar toxic effect of alcohol or through deficit of folic acid and, eventually, B12, and normalizes after 2-4 months of abstinence. [20] The MCV might also be increased by the direct toxic action of organic compounds (especially benzene). The same principle applied in the glycosylated haemoglobin test for estimation of glycaemia variation was developed on the basis that plasma acetaldehyde increases after alcohol consumption, and binds to plasma proteins, including haemoglobin. Therefore whole bloodassociated acetaldehyde assay (WBAA) estimates the alcohol consumption during the last 3 months. Fatty acid ethyl esters measured in the hair could also be a quantifier marker for chronic exposure. These tests are only utilised in research studies at the moment and have not been validated as clinical diagnostic tests. Because all these biomarkers lack sufficient sensitivity and specificity, proteomics techniques will be able to validate biomarker panels. There are promising attempts in this respect: for example, in liver steatosis induced by alcohol consumption, a down-regulation of alcohol dehydrogenase and an up-regulation of aldehyde dehydrogenase were seen as well as changes in the levels of methionine metabolizing enzymes. [21] The dissociation between occupational and nonoccupational factors is seldom difficult and becomes impossible when toxicants are identical. A detailed occupational anamnesis is an important tool when alcohol use and/or abuse is inconsistent. Therefore, we consider, in this particular case, there are more arguments for occupational induced hepatopathy and neuropathy than for alcohol as etiological factor.

\section{Conclusions.}

It is impossible to distinguish between occupational toxic hepatitis and alcohol induced hepatitis by current routine investigations.

An occupational medicine consultation, workplace risk evaluation and early identification of biological markers of occupational exposure shouldn't be delayed, especially when anamnesis suggests organic solvents exposure.

\section{References:}

1. International Programme on Chemical Safety and the European Commission (C) IPCS 20042012. Benzene. from http://www.ilo.org/dyn/ icsc/showcard.display?p card id $=0015$

2. Lundqvist, G., Flodin, U. \& Axelson, O. (1999). A case-control study of fatty liver disease and organic solvent exposure. Am J Ind Med. 35(2), 132-136..

3. Malaguarnera, G., Cataudella, E., Giordano, M., Nunnari, G., Chisari, G. \& Malaguarnera, M. (2012). Toxic hepatitis in occupational exposure to solvents. World J Gastroenterol. 18(22), 27562766. doi: 10.3748/wjg.v18.i22.2756.

4. Brodkin, C.A., Daniell, W., Checkoway, H., Echeverria, D., Johnson, J., Wang, K., Sohaey, R., Green, D., Redlich, C., Gretch, D. \& et al. (1995). Hepatic ultrasonic changes in workers exposed to perchloroethylene. Occup Environ Med. 52(10), 679-685..

5. McIntyre, A.S. \& Long, R.G. (1992). Fatal 
fulminant hepatic failure in a ,solvent abuser'. Postgrad Med J. 68(795), 29-30

6. D'Andrea, M.A. \& Reddy, G.K. (2014). Health risks associated with crude oil spill exposure. $\mathrm{Am}$ $J$ Med. 127(9), 886 e889 -813. doi: 10.1016/j. amjmed.2014.04.035.

7. Carvalho, F.M., Silvany Neto, A.M., Mendes, J.L., Cotrim, H.P., Nascimento, A.L., Lima Junior, A.S. \& Cunha, T.O. (2006). [Liver enzyme abnormalities among oil refinery workers]. Rev Saude Publica. 40(1), 92-98. doi: / S0034-89102006000100015.

8. Mohammadi, S., Mehrparvar, A., Labbafinejad, Y. \& Attarchi, M.S. (2010). The effect of exposure to a mixture of organic solvents on liver enzymes in an auto manufacturing plant. Journal of Public Health. 18(6), 553-557. doi: 10.1007/s10389010-0340-z.

9. Arndt, T. (2001). Carbohydrate-deficient transferrin as a marker of chronic alcohol abuse: a critical review of preanalysis, analysis, and interpretation. Clin Chem. 47(1), 13-27.

10. Neumann, T. \& Spies, C. (2003). Use of biomarkers for alcohol use disorders in clinical practice. Addiction. 98 Suppl 2, 81-91..

11. Helander, A., Bottcher, M., Fehr, C., Dahmen, N. $\&$ Beck, O. (2009). Detection times for urinary ethyl glucuronide and ethyl sulfate in heavy drinkers during alcohol detoxification. Alcohol Alcohol. 44(1), 55-61. doi: 10.1093/alcalc/ agn084.

12. Peterson, K. (2004/2005). Biomarkers for Alcohol Use and Abuse - A summary. Alcohol Research \& Health. 28(1), 30-37.

13. Niemela, O. (2016). Biomarker-Based Approaches for Assessing Alcohol Use Disorders. Int J Environ Res Public Health. 13(2), 166. doi: 10.3390/ijerph13020166

14. Petrariu, F., Alexinnchi, O., Chirita, R., Chirita, V., Ciobica, A., Padurariu, M., Lefter, R., Dobrin, R., Popescu, R., Anton, E., Arcan, O. \& Timofte, D. (2014). The dynamics of some oxidative stress markers in 3, 6 and 12-months alcohol abstinent patients: possible relevance for the usage of antioxidants in alcohol withdrawal. Romanian Journal of Laboratory Medicine. 22(4), 451-457. doi: 10.2478/rrlm-2014-0040.
15. Skowron, J., Miranowicz-Dzierzawska, K., Zapor, L., Golofit-Szymczak, M. \& Starek, A. (2001). Interactions of some organic solvents: hydrocarbons and chloroalkene. Int J Occup Saf Ergon. 7(1), 35-47. doi: 10.1080/10803548.200 1.11076475.

16. Chang, W.J., Joe, K.T., Park, H.Y., Jeong, J.D. \& Lee, D.H. (2013). The relationship of liver function tests to mixed exposure to lead and organic solvents. Ann Occup Environ Med. 25(1), 5. doi: 10.1186/2052-4374-25-5

17. Ernstgard, L., Gullstrand, E., Johanson, G. \& Lof, A. (1999). Toxicokinetic interactions between orally ingested chlorzoxazone and inhaled acetone or toluene in male volunteers. Toxicol Sci. 48(2), 189-196

18. Johns, D.O., Daniell, W.E., Shen, D.D., Kalman, D.A., Dills, R.L. \& Morgan, M.S. (2006). Ethanol-induced increase in the metabolic clearance of 1,1,1-trichloroethane in human volunteers. Toxicol Sci. 92(1), 61-70. doi: $10.1093 /$ toxsci/kfj210.

19. Traphagen, N., Tian, Z. \& Allen-Gipson, D. (2015). Chronic Ethanol Exposure: Pathogenesis of Pulmonary Disease and Dysfunction. Biomolecules. 5(4), 2840-2853. doi: 10.3390/ biom5042840

20. H Hannuksela, M.L., Liisanantti, M.K., Nissinen, A.E. \& Savolainen, M.J. (2007). Biochemical markers of alcoholism. Clin Chem Lab Med. 45(8), 953-961. doi: 10.1515/CCLM.2007.190

21. Fernando, H., Wiktorowicz, J.E., Soman, K.V., Kaphalia, B.S., Khan, M.F. \& Shakeel Ansari, G.A. (2013). Liver proteomics in progressive alcoholic steatosis. Toxicol Appl Pharmacol. 266(3), 470-480. doi: 10.1016/j.taap.2012.11.017 